\title{
Preparation and characterization of antithrombogenic chitosan/alginate films with enhanced physical stability by cross-linking using layer-by- layer method
}

\author{
Kyu-Hong Kyung ${ }^{1}$, Sae-Hoon $\mathrm{Kim}^{2}$, Seimei Shiratori ${ }^{1}$ \\ ${ }^{1}$ School of Integrated Design Engineering, Keio University, Yokohama 223-8522, Japan \\ ${ }^{2}$ Department of Ceramic Engineering, Kangnung-wonju National University, 123 Jibyeon-dong, Kangwon-do 210-702, \\ Korea
}

E-Mail address : kyong_kyuhong@appi2.appi.keio.ac.jp

\begin{abstract}
We successfully fabricated (chitosan/alginate) (CHI/ALG) multilayer thin film by layer-by-layer (LBL) method. Film durability was enhanced by forming cross-linking using heat treatment, glutaraldehyde, and calcium chloride. Indeed, the adsorption of fibrinogen onto CHI/ALG multilayer thin film was determined by QCM system and it was found that physical stability and protein resistance of CHI/ALG films were improved by cross-linking of the films.
\end{abstract}

\section{INTRODUCTION}

Polyelectrolyte layer-by-layer (LBL) self-assembled biointerfaces have attracted great interest in recent years due to their prominent advantages [1-7]. It is based on the alternate adsorption of oppositely charged materials in aqueous solutions via electrostatic attraction. It has many advantages such as a simple process, water-based roomtemperature deposition at normal pressure, no limit of thickness, and no need for complicated equipment. In addition, the thickness of the thin films can be controlled with nanoscale accuracy [8-9].

On the one hand, chitosan (CHI) has been especially interesting as it is the only positively charged polysaccharide that is readily available. $\mathrm{CHI}$ is a biodegradable, non-toxic and biocompatible material with interesting properties for use in tissue engineering and other biomedical applications [10-13]. Alginate (ALG) has been widely used in the form of hydrogels for many biomedical applications, including as scaffolds for tissue engineering, delivery vehicles for drugs and as model extracellular matrices for fundamental biological studies $[14,15]$.

In this point of view, combinations of CHI and ALG have been widely used as drug delivery systems, where ALG beads are coated with CHI. Cross-linking is a strategy for modifying the properties of multilayer films that can have profound influence on different properties, including the stiffness, wettability and the degradation profile of the thin film, and on the adhesion of different types of cells [16].
CHI/ALG multilayer film can offer the unique properties for immobilization of antibody with retaining its binding activity. Although the self-assembled multilayer film of chitosan and alginate has been reported, to our knowledge, there is no report to study its assembly kinetics, tunability, mechanism, and antibody immobilization via the adjustment of assembly $\mathrm{pH}$, all of which are important for construction of excellent biointerfaces in different applications $[1,17]$

In this study, we enhanced physical stability of (CHI/ALG) film by creating cross-linking using heat treatment, glutaraldehyde, and calcium chloride. And we fabricated an antithrombogenic thin film using LBL method in order to produce adhesive films.

\section{Materials and Methods}

\subsection{Materials}

Chitosan was obtained from wako as positive charge solution. Sodium alginate was obtained from nacalai tesque (Kyoto, Japan) as negative charge solution. CHI and ALG solutions were prepared with a concentration of $2 \mathrm{mg} / \mathrm{mL}$ in $\mathrm{NaCl}$. The $\mathrm{pH}$ of both solutions was adjusted by adding acetic acid. Glutaraldehyde (GLUT) and calcium chloride $\left(\mathrm{CaCl}_{2}\right)$ were chosen as the water soluble cross-linker for CHI and ALG. GLUT and $\mathrm{CaCl}_{2}$ solution with $0.2 \mathrm{wt} \%$ and $0.1 \mathrm{M}$ were prepared, respectively. 


\subsection{Preparation of thin films}

The QCM substrates were ultrasonically agitated in $\mathrm{KOH}$ solution (1.0 wt\%) mixed with ultrapure water and ethanol (2:3 in volume ratio) for $5 \mathrm{~min}$ and then rinsed with ultrapure water. By this treatment, negatively charged substrates were prepared. Then, we assembled $(\mathrm{PDDA} / \mathrm{PSS})_{3}$ bilayers thin film on the electrode of QCM as a buffer layer in order to remove the influence of surface state of QCM.

The substrates were immersed in cationic CHI solution and then rinsed three times in pure water for 1 min each. The positively charged substrates produced by the deposition of $\mathrm{CHI}$ were subsequently immersed in the negatively charged ALG solution and then the same rinsing procedure (three times for 1 min each) was carried out. This coating sequence was automatically repeated up to 10 bilayers without stopping using an automatic dipping machine. Hereafter, when we deposit the material $\mathrm{B}$ over $\mathrm{A}$, we describe the system as $(\mathrm{A} / \mathrm{B})$ and when we repeat this sequence 10 times, we describe it as $(\mathrm{A} / \mathrm{B})_{10}$.

\subsection{Characterization of thin films}

Surface morphology was investigated by fieldemission scanning electron microscopy (FE-SEM; Hitachi S-4700), and the root mean square (RMS) surface roughness of films was measured by atomic force microscopy (AFM; Digital Instrument Nanoscope IIIa) in the tapping mode. Before and after cross-linking of $(\mathrm{CHI} / \mathrm{ALG})_{10}$ films, they were investigated by in situ FTIR spectroscopy with a ALPHA-T, Bruker. The thickness of the prepared films was measured by ellipsometry (ULVAC ESM-1A). Protein adsorption was determined by QCM device when (CHI/ALG) films were contacted with fibrinogen solution at room temperature for $1 \mathrm{~h}$. The fibrinogen was diluted to a desired concentration of 0.5 $\mathrm{mg} / \mathrm{ml}$ in PBS $(\mathrm{pH}=7.4)$.

\section{Results and Discussion}

\subsection{Characterization of film surface}

The AFM image of the thin films was depicted in fig. 1.As shown in this fig. 1, clusters agglomerated and the average size of the clusters was affected by the $\mathrm{pH}$ of ALG solution.

The surface roughness of the (CHI/ALG) thin films (a, $\mathrm{b}$, and c) were ca. 5.1, 4.2, and $3.4 \mathrm{~nm}$, respectively. These figures show that the surface roughness increased with the decrease $\mathrm{pH}$ of ALG solution.

The thicknesses of the (CHI/ALG) films were regularly increased by increasing the number of bilayers. The average thicknesses of (CHI/ALG) films were determined to be ca. $1.4 \mathrm{~nm}$. This means that the thickness of the films was well controlled by the LBL method. To make the films thicker, we added $\mathrm{NaCl}$ to the chitosan and alginate solution. As shown in figure 2, clusters agglomerated and the average size of the clusters was affected by the amount of $\mathrm{NaCl}$ added. By adding $\mathrm{NaCl}(0.1 \mathrm{M})$ to $\mathrm{CHI}$ and ALG solutions, the thickness of the thin films was increased from 15 to ca. $39 \mathrm{~nm}$ (ca. 2.5 -fold).
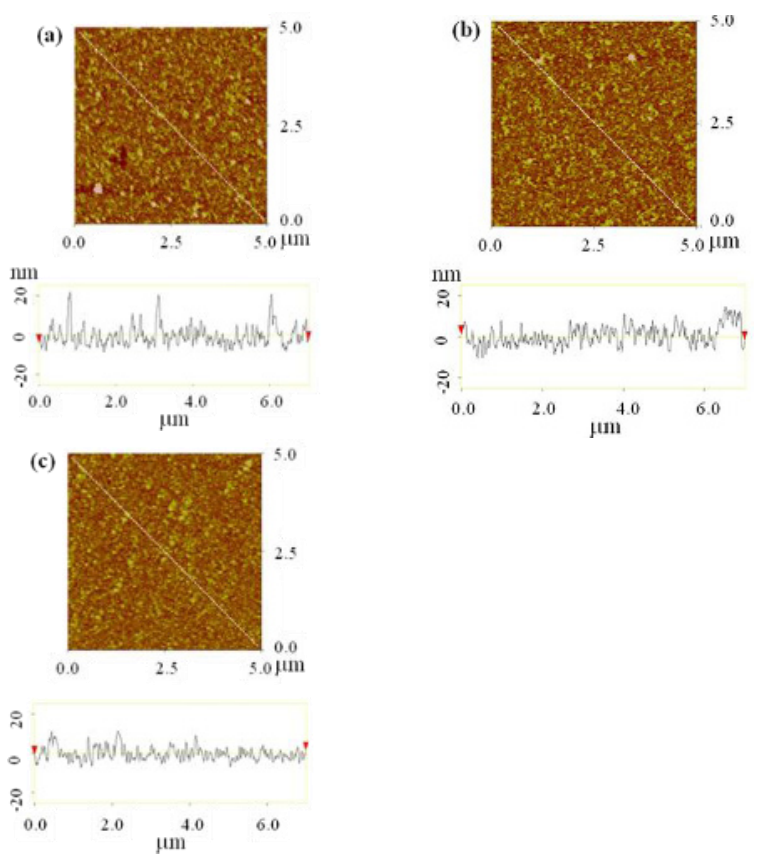

Fig. 1. AFM(top view; $5 \times 5 \mu \mathrm{m}^{2}$ ) images as a function of $\mathrm{pH}$ of alginate. The assembly $\mathrm{pH}$ of chitosan of all these films is 4.0 . The assembly $\mathrm{pH}$ of alginate is (a) 4.0, (b) 5.0, (c) 6.0.
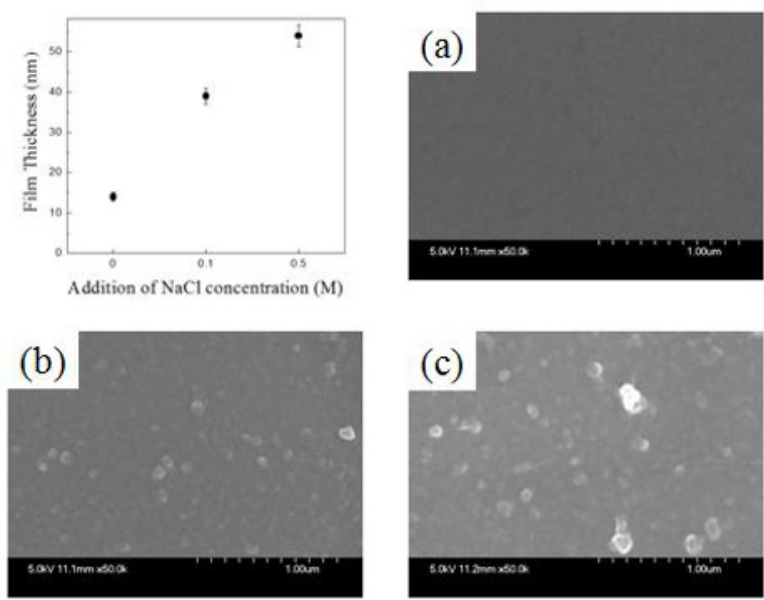

Fig.2. Thickness and FE-SEM image of (CHI4.0/ALG5.0) $)_{10}$ film as a function of the amount of $\mathrm{NaCl}$ added to $\mathrm{CHI}$ and ALG solution: (a) $0 \mathrm{M}$, (b) $0.1 \mathrm{M}$, and (c) $0.5 \mathrm{M} \mathrm{NaCl}$ added to $\mathrm{CHI}$ and ALG.

\subsection{In situ cross-linking of chitosan/alginate films}

Cross-linking was carried out to increase the stability of (CHI/ALG) film. In this experiment, heat treatment, GLUT and $\mathrm{CaCl}_{2}$ were chosen as the water soluble crosslinker for CHI and ALG. As shown in Table I, the thickness of non-cross-linked (CHI/ALG) film was decreased from 39 to $32.5 \mathrm{~nm}$ after 1 hour dipping into fibrinogen PBS solution. The thickness values were masured by ellipsometry. However, all of the three crosslinked (CHI/ALG) films (heat treatment, GLUT treatment and $\mathrm{CaCl}_{2}$ treatment) thickness was not change. 
Therefore, it was demonstrated that Cross-linking (CHI/ALG) films enhanced the stability in the PBS solution.

Table 1. Change of thickness of (CHI/ALG $)_{10}$ film before and after adsorption of fibrinogen.

\begin{tabular}{c|c|c}
\hline \multirow{2}{*}{$\begin{array}{c}\text { Cross-linking } \\
\text { method }\end{array}$} & $\begin{array}{c}\text { Before fibrinogen } \\
\text { adsorption }\end{array}$ & $\begin{array}{c}\text { After fibrinogen } \\
\text { adsorption }\end{array}$ \\
\cline { 2 - 3 } $\begin{array}{c}\text { Without } \\
\text { cross-linking }\end{array}$ & 39 & 32.5 \\
\hline $\begin{array}{c}\text { Heat treatment } \\
\left(180^{\circ} \mathrm{C}\right)\end{array}$ & 32 & 32 \\
\hline Glutaraldehyde & 39.5 & 39 \\
\hline Calcium chloride & 37 & 36.5 \\
\hline
\end{tabular}

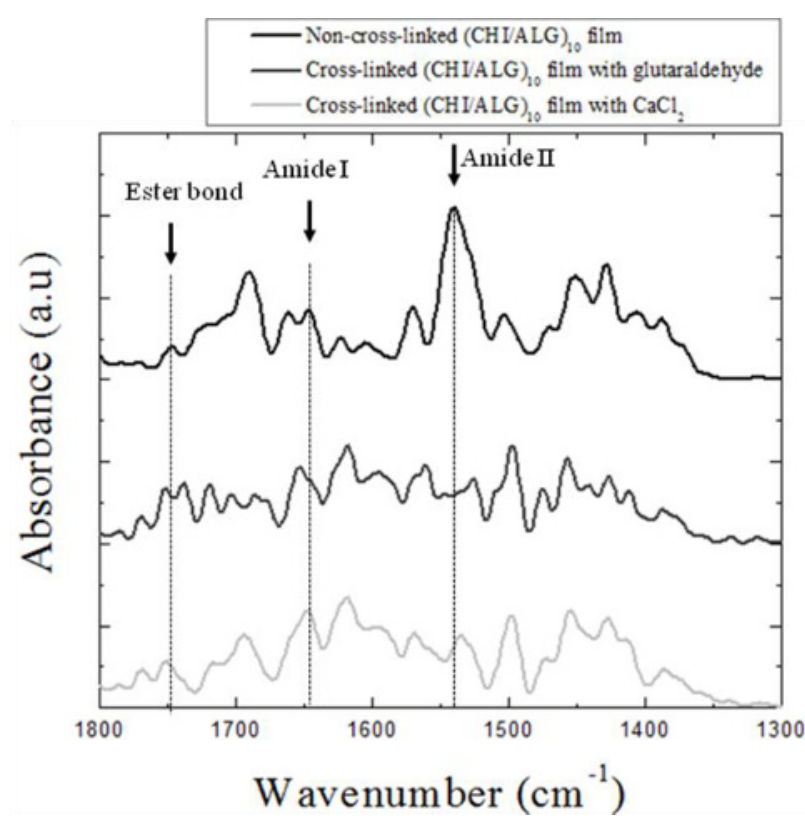

Fig. 3. FT-IR spectra of non-cross-linked and cross-linked (CHI/ALG) film.

As shown in Fig.3, there is decrease of the amide II peaks of CHI and a slight increase of amide I and ester band. The saccharide peaks also appear slightly affected by the GLUT and $\mathrm{CaCl}_{2}$ cross-linking, however with only small peak variations.

\subsection{Protein adsorption}

The surface of $(\mathrm{CHI} / \mathrm{ALG})_{10}$ films before and after the adsorption of fibrinogen were observed by FE-SEM. The results were shown in Fig.4. In this figure, the control films, the films treated by GLUT, and the films treated by $\mathrm{CaCl}_{2}$ were compared. The left side of the images (a), (c), and (e) are the images before the adsorption, and (b), (d) and (f) are the images after the adsorption.

As shown in the Fig.4 it seems that the cross-linked (CHI/ALG) film with $\mathrm{CaCl}_{2}$ is much higher bloodcompatibility than cross-linked (CHI/ALG) film with GLUT because the former forms hydrogel, which showed antithrombogenecity. The formation of thrombi is evident on surface without (CHI/ALG) coating for fibrinogen which show large aggregates and extension. In the case of cross-linked (CHI/ALG) films, otherwise, the fibrinogen adsorption not cover on the surface.
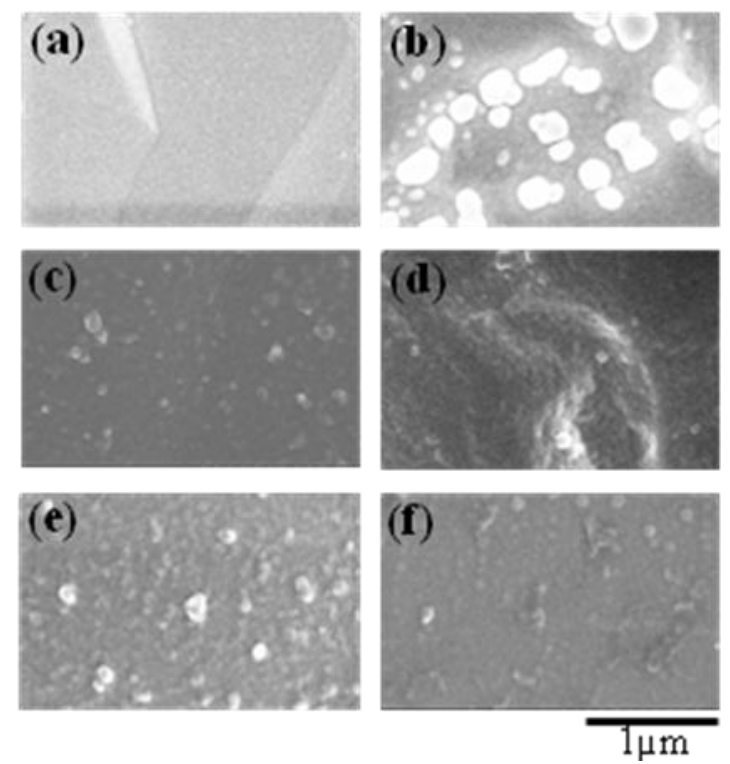

Fig.4. FE-SEM images of (CHI/ALG $)_{10}$ films before fibrinogen adsorption (a) control, (c) cross-linked (CHI/ALG) 10 with glutaraldehyde, (e) cross-linked (CHI/ALG) $)_{10}$ with calcium chloride and after fibrinogen adsorption (b) control, (d) crosslinked (CHI/ALG) $)_{10}$ with glutaraldehyde, (f) cross-linked $(\mathrm{CHI} / \mathrm{ALG})_{10}$ with calcium chloride.

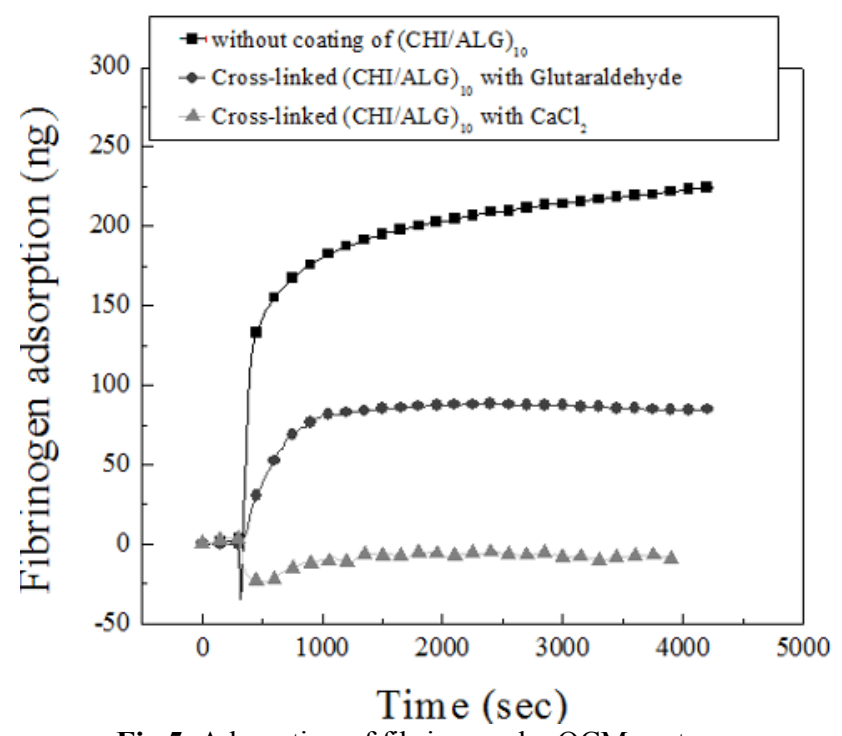

Fig.5. Adsorption of fibrinogen by QCM system.

As shown in Fig.5, the protein adsorption was determined by QCM device. QCM measurement demonstrated that fibrinogen adsorption was less on cross-linked films as compared to that on non-crosslinked films. As the result, the lowest adsorption of fibrinogen is obtained in the case of the cross-linked (CHI/ALG) film with $\mathrm{CaCl}_{2}$.

ALG is a chain-forming polyscaccharide, which can be cross-linked by complexation of its carboxylic groups with $\mathrm{Ca}^{2+}$, thus production mechanically stable hydrogels. 


\section{Conclusion}

Film durability of (CHI/ALG) film was enhanced by increasing its physical stability by forming cross-linking using heat treatment, glutaraldehyde, and calcium chloride. Then, the cross-linked (CHI/ALG) film showed antithrombogenicity. LBL assembled multilayer film can thus be considered an attractive approach to incorporate biomolecules into the surface of biomaterials. It can be expected that this anti-thrombogenicity will be widely applied for biomedical devices in contact with blood.

\section{Acknowledgment}

This work was supported by the National Research Foundation of Korea Grant funded by the Korean Government (NRF - 2012 - 013 - D00062)

\section{References}

1. W. Yuan, H. Dong, C. M. Li, X. Cui, L. Yu, Z. Lu, Q. Zhou, Langmuir 23 (2007) 13046-13052

2. D. Yoo, S. S. Shiratori, M. F. Rubner, Macromolecules 31 (1998) 4309-4318.

3. S. Shiratori, M. F. Rubner, Macromolecules 33 (2000) 4213-4219.

4. B. Thierry, F. M. Winnik, Y. Merhi, J. Silver, M. Tabrizian, Biomacromolecules 4 (2003) 1564-1571.

5. T. C. Wang, M. F. Rubner, R. E. Cohen, Langmuir 18 (2002) 3370-3375.

6. Y. Liu, Y. Wang, H. Lu, R. O. J. Claus, Phys. Chem. B 103 (1999) 2035-2036.

7. M. K. Park, S. Deng, R. C. Advincula, J. Am. Chem. Soc. 126 (2004) 13723-13731.

8. G. Decher, Science 277 (1997) 1232-1237.

9. K. H. Kyung, K. Fujimoto, S. Shiratori, J. H. Kim, S. H. Kim, Jap. J. Appl. Phys. 49 (2010) 045001.

10. N. M. Alves, C. Picart, J. F. Mano, Macromol. Biosci. 9 (2009) 776-785.

11. S. V. Madihally, H. W. T. Matthew, Biomaterials 20 (1999) 1133-1142.

12. J. K. F. Suh, H. W. T. Matthew, Biomaterials 21 (2000) 2589.

13. A. Di Martino, M. Sittinger, M. V. Risbud, Biomaterials 26 (2005) 5983.

14. J. A. Rowley, G. Madlambayan, D. J. Money, Biomaterials 20 (1999) 45.

15. A. D. August, H. J. Kong, D. J. Mooney, Macromol. Biosci. 6 (2006) 623-633.

16. N. M. Alves, C. Picrt, J. F. Mano, Macromol. Biosci. 9 (2009) 776-785.

17. S. Ye, C. Wang, X. Liu, Z. Tong, B. Ren, F. J. Zeng, Controlled Release 112 (2006) 79-87. 